\title{
Solitons of the midpoint mapping and affine curvature
}

\author{
Christine Rademacher and Hans-Bert Rademacher(e)
}

\begin{abstract}
For a polygon $x=\left(x_{j}\right)_{j \in \mathbb{Z}}$ in $\mathbb{R}^{n}$ we consider the midpoints polygon $(M(x))_{j}=\left(x_{j}+x_{j+1}\right) / 2$. We call a polygon a soliton of the midpoints mapping $M$ if its midpoints polygon is the image of the polygon under an invertible affine map. We show that a large class of these polygons lie on an orbit of a one-parameter subgroup of the affine group acting on $\mathbb{R}^{n}$. These smooth curves are also characterized as solutions of the differential equation $\dot{c}(t)=B c(t)+d$ for a matrix $B$ and a vector $d$. For $n=2$ these curves are curves of constant generalized-affine curvature $k_{g a}=k_{g a}(B)$ depending on $B$ parametrized by generalized-affine arc length unless they are parametrizations of a parabola, an ellipse, or a hyperbola.
\end{abstract}

Mathematics Subject Classification. 51M04 (15A16 53A15).

Keywords. Discrete curve shortening, polygon, affine mappings, soliton, midpoints polygon, linear system of ordinary differential equations.

\section{Introduction}

We consider an infinite polygon $\left(x_{j}\right)_{j \in \mathbb{Z}}$ given by its vertices $x_{j} \in \mathbb{R}^{n}$ in an $n$-dimensional real vector space $\mathbb{R}^{n}$ resp. an $n$-dimensional affine space $\mathbb{A}^{n}$ modelled after $\mathbb{R}^{n}$. For a parameter $\alpha \in(0,1)$ we introduce the polygon $M_{\alpha}(x)$ whose vertices are given by

$$
\left(M_{\alpha}(x)\right)_{j}:=(1-\alpha) x_{j}+\alpha x_{j+1} .
$$

For $\alpha=1 / 2$ this defines the midpoints polygon $M(x)=M_{1 / 2}(x)$. On the space $\mathcal{P}=\mathcal{P}\left(\mathbb{R}^{n}\right)$ of polygons in $\mathbb{R}^{n}$ this defines a discrete curve shortening process $M_{\alpha}: \mathcal{P} \longrightarrow \mathcal{P}$, already considered by Darboux [4] in the case of a closed resp.

We are grateful to the referee for his suggestions. 


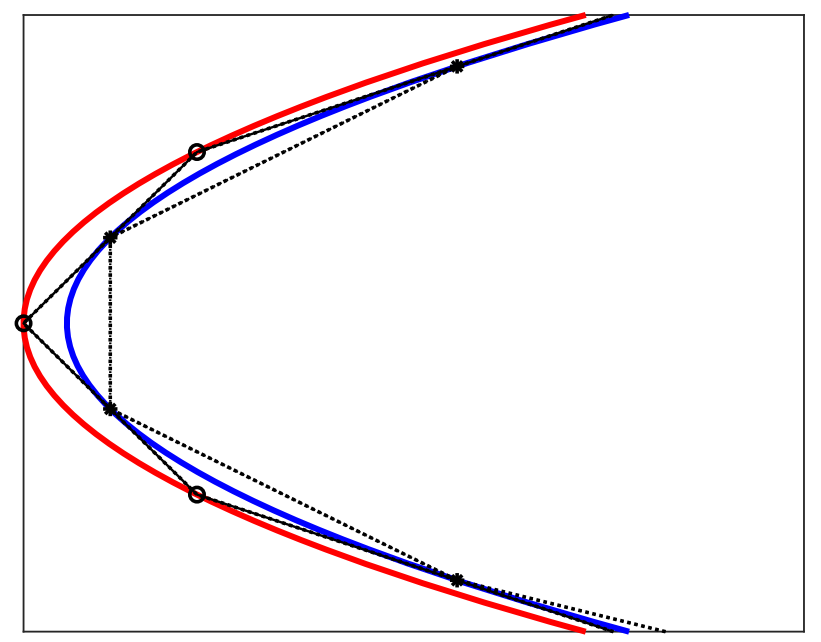

Figure 1 The parabola $c(t)=\left(t^{2} / 2, t\right)$ as soliton of the midpoints map $M$

periodic polygon. For a discussion of this elementary geometric construction see Berlekamp et al. [1].

The mapping $M_{\alpha}$ is invariant under the canonical action of the affine group. The affine group $\operatorname{Aff}(n)$ in dimension $n$ is the set of affine maps $(A, b): \mathbb{R}^{n} \longrightarrow$ $\mathbb{R}^{n}, x \longmapsto A x+b$. Here $A \in \operatorname{Gl}(n)$ is an invertible matrix and $b \in \mathbb{R}^{n}$ a vector. The translations $x \longmapsto x+b$ determined by a vector $b$ form a subgroup isomorphic to $\mathbb{R}^{n}$. Let $\alpha \in(0,1)$. We call a polygon $x_{j}$ a soliton for the process $M_{\alpha}$ (or affinely invariant under $M_{\alpha}$ ) if there is an affine map $(A, b) \in \operatorname{Aff}(n)$ such that

$$
\left(M_{\alpha}(x)\right)_{j}=A x_{j}+b
$$

for all $j \in \mathbb{Z}$. In Theorem 1 we describe these solitons explicitly and discuss under which assumptions they lie on the orbit of a one-parameter subgroup of the affine group acting canonically on $\mathbb{R}^{n}$. We call a smooth curve $c: \mathbb{R} \longrightarrow \mathbb{R}^{n}$ a soliton of the mapping $M_{\alpha}$ resp. invariant under the mapping $M_{\alpha}$ if there is for some $\epsilon>0$ a smooth mapping $s \in(-\epsilon, \epsilon) \longmapsto(A(s), b(s)) \in \operatorname{Aff}(n)$ such that for all $s \in(-\epsilon, \epsilon)$ and $t \in \mathbb{R}$ :

$$
\tilde{c}_{s}(t):=(1-\alpha) c(t)+\alpha c(t+s)=A(s) c(t)+b(s) .
$$

Then for some $t_{0} \in \mathbb{R}$ and $s \in(-\epsilon, \epsilon)$ the polygon $x_{j}=c\left(j s+t_{0}\right), j \in \mathbb{Z}$ is a soliton of $M_{\alpha}$. The parabola is an example of a soliton of $M=M_{1 / 2}$, cf. Fig. 1 and Example 1, Case (e). We show in Theorem 2 that the smooth curves invariant under $M_{\alpha}$ coincide with the orbits of a one-parameter subgroup of the affine group $\operatorname{Aff}(n)$ acting canonically on $\mathbb{R}^{n}$. For $n=2$ we give a characterization of these curves in terms of the general-affine curvature in Sect. 5 . 
The authors discussed solitons, i.e. curves affinely invariant under the curve shortening process $T: \mathcal{P}\left(\mathbb{R}^{n}\right) \longrightarrow \mathcal{P}\left(\mathbb{R}^{n}\right)$ with

$$
(T(x))_{j}=\frac{1}{4}\left\{x_{j-1}+2 x_{j}+x_{j+1}\right\}
$$

in [9]. The solitons of $M=M_{1 / 2}$ form a subclass of the solitons of $T$, since $(T(x))_{j}=\left(M^{2}(x)\right)_{j-1}$. Instead of the discrete evolution of polygons one can also investigate the evolution of polygons under a linear flow, cf. Viera and Garcia [11] and [9, Sec. 4] or a non-linear flow, cf. Glickenstein and Liang [5].

\section{The affine group and systems of linear differential equations of first order}

The affine group $\operatorname{Aff}(n)$ is a semidirect product of the general linear group $\mathrm{Gl}(n)$ and the group $\mathbb{R}^{n}$ of translations. There is a linear representation

$$
(A, b) \in \operatorname{Aff}(n) \longrightarrow\left(\begin{array}{c|c}
A \mid b \\
\hline 0 & 1
\end{array}\right) \in \mathrm{Gl}(n+1),
$$

of the affine group in the general linear group $\mathrm{Gl}(n+1)$, cf. [8, Sec. 5.1]. We use the following identification

$$
\left(\begin{array}{c|c}
A & b \\
\hline 0 & 1
\end{array}\right)\left(\frac{x}{1}\right)=\left(\frac{A x+b}{1}\right) .
$$

Hence we can identify the image of a vector $x \in \mathbb{R}^{n}$ under the affine map $x \longmapsto A x+b$ with the image $\left(\frac{A x+b}{1}\right)$ of the extended vector $\left(\frac{x}{1}\right)$. Using this identification we can write down the solution of an inhomogeneous system of linear differential equations with constant coefficients using the power series $F_{B}(t)$ which we introduce now:

Proposition 1. For a real $(n, n)$-matrix $B \in M_{\mathbb{R}}(n)$ we denote by $F_{B}(t) \in$ $M_{\mathbb{R}}(n)$ the following power series:

$$
F_{B}(t)=\sum_{k=1}^{\infty} \frac{t^{k}}{k !} B^{k-1} .
$$

(a) We obtain for its derivative:

$$
\frac{d}{d t} F_{B}(t)=\exp (B t)=B F_{B}(t)+\mathbb{1}
$$

The function $F_{B}(t)$ satisfies the following functional equation:

$$
F_{B}(t+s)=F_{B}(s)+\exp (B s) F_{B}(t),
$$

resp. for $j \in \mathbb{Z}, j \geq 1$ :

$$
\begin{aligned}
F_{B}(j) & =\{\mathbb{1}+\exp (B)+\exp (2 B)+\cdots+\exp ((j-1) B)\} F_{B}(1) \\
& =(\exp (B)-\mathbb{1})^{-1}(\exp (j B)-\mathbb{1}) F_{B}(1) .
\end{aligned}
$$


(b) The solution $c(t)$ of the inhomogeneous system of linear differential equations

$$
\dot{c}(t)=B c(t)+d
$$

with constant coefficients (i.e. $B \in M_{\mathbb{R}}(n, n), d \in \mathbb{R}^{n}$ ) and with initial condition $v=c(0)$ is given by:

$$
c(t)=v+F_{B}(t)(B v+d)=\exp (B t)(v)+F_{B}(t)(d) .
$$

Proof. (a) Equation (6) follows immediately from Eq. (5). Then we compute

$$
\frac{d}{d t}\left(F_{B}(t+s)-\exp (B s) F_{B}(t)\right)=\exp (B(t+s))-\exp (B s) \exp (B t)=0 .
$$

Since $F_{B}(0)=0$ Eq. (7) follows. And this implies Eq. (8).

(b) We can write the solution of the differential equation (8)

$$
\frac{d}{d t}\left(\frac{c(t)}{1}\right)=\left(\begin{array}{c|c}
B & d \\
\hline 0 & 0
\end{array}\right)\left(\frac{c(t)}{1}\right)
$$

as follows:

$$
\begin{aligned}
\left(\frac{c(t)}{1}\right) & =\exp \left(\left(\frac{B}{0} \mid \begin{array}{l}
0 \\
\hline 0
\end{array}\right)\left(\frac{v}{1}\right)\right. \\
& =\left(\begin{array}{c|c}
\exp (B t) & F_{B}(t)(d) \\
\hline 0 & 1
\end{array}\right)\left(\frac{v}{1}\right)=\left(\frac{\exp (B t)(v)+F_{B}(t)(d)}{1}\right)
\end{aligned}
$$

which is Eq. (9). One could also differentiate Eq. (9) and use Eq. (6)

Remark 1. Equation (2) shows that $c(t)$ is the orbit

$$
t \in \mathbb{R} \longmapsto c(t)=\exp \left(\left(\begin{array}{c|c}
B \mid d \\
\hline 0 & 0
\end{array}\right) t\right)\left(\frac{v}{1}\right) \in \mathbb{R}^{n} .
$$

of the one-parameter subgroup

$$
t \in \mathbb{R} \longmapsto \exp \left(\left(\begin{array}{c|c}
B \mid d \\
\hline 0 & 0
\end{array}\right) t\right) \in \operatorname{Aff}(n)
$$

of the affine group $\operatorname{Aff}(n)$ acting canonically on $\mathbb{R}^{n}$.

\section{Polygons invariant under $M_{\alpha}$}

Theorem 1. Let $(A, b): x \in \mathbb{R}^{n} \longmapsto A x+b \in \mathbb{R}^{n}$ be an affine map and $v \in \mathbb{R}^{n}$. Assume that for $\alpha \in(0,1)$ the value $1-\alpha$ is not an eigenvalue of $A$, i.e. the matrix $A_{\alpha}:=\alpha^{-1}(A+(\alpha-1) \mathbb{1})$ is invertible. Then the following statements hold:

(a) There is a unique polygon $x \in \mathcal{P}\left(\mathbb{R}^{n}\right)$ with $x_{0}=v$ which is a soliton for $M_{\alpha}$ resp. affinely invariant under the mapping $M_{\alpha}$ with respect to the affine map $(A, b)$, cf. Eq. (1). If $b_{\alpha}=\alpha^{-1} b$, then for $j>0$ :

$$
\begin{aligned}
x_{j} & =A_{\alpha}^{j}(v)+A_{\alpha}^{j-1}\left(b_{\alpha}\right)+\cdots+A_{\alpha}\left(b_{\alpha}\right)+b_{\alpha} \\
& =v+\left(A_{\alpha}^{j}-\mathbb{1}\right)\left(v+\left(A_{\alpha}-\mathbb{1}\right)^{-1}\left(b_{\alpha}\right)\right) .
\end{aligned}
$$


and for $j<0$ :

$$
\begin{aligned}
x_{j} & =A_{\alpha}^{j}(v)-A_{\alpha}^{j}\left(b_{\alpha}\right)+\cdots+A_{\alpha}^{-1}\left(b_{\alpha}\right) \\
& =v+\left(A_{\alpha}^{j}-\mathbb{1}\right)\left(v-\left(A_{\alpha}^{-1}-\mathbb{1}\right)^{-1}\left(A_{\alpha}^{-1}\left(b_{\alpha}\right)\right) .\right.
\end{aligned}
$$

(b) If $A_{\alpha}=\exp \left(B_{\alpha}\right)$ for a $(n, n)$-matrix $B_{\alpha}$ and if $b_{\alpha}=F_{B_{\alpha}}(1)\left(d_{\alpha}\right)$ for $a$ vector $d_{\alpha} \in \mathbb{R}^{n}$ then the polygon $x_{j}$ lies on the smooth curve

$$
c(t)=v+F_{B_{\alpha}}(t)\left(B_{\alpha} v+d_{\alpha}\right)
$$

i.e. $x_{j}=c(j)$ for all $j \in \mathbb{Z}$.

Proof. (a) By Eq. (1) we have

$$
(1-\alpha) x_{j}+\alpha x_{j+1}=A x_{j}+b
$$

for all $j \in \mathbb{Z}$. Hence the polygon is given by $x_{0}=v$ and the recursion formulae

$$
x_{j+1}=A_{\alpha}\left(x_{j}\right)+b_{\alpha} ; x_{j}=A_{\alpha}^{-1}\left(x_{j+1}-b_{\alpha}\right) .
$$

for all $j \in \mathbb{Z}$. Then Eqs. (11) and (12) follow.

(b) For $A_{\alpha}=\exp B_{\alpha} ; b_{\alpha}=F_{B_{\alpha}}\left(d_{\alpha}\right)$ we obtain from Eq. (6) for all $j \in \mathbb{Z}$ : $A_{\alpha}-\mathbb{1}=B_{\alpha} F_{B_{\alpha}}(1)$ and $A_{\alpha}^{j}-\mathbb{1}=B_{\alpha} F_{B_{\alpha}}(j)$. Hence for $j>0$ :

$$
\begin{aligned}
x_{j} & =v+\left(A_{\alpha}^{j}-\mathbb{1}\right)\left(v+\left(A_{\alpha}-\mathbb{1}\right)^{-1} b_{\alpha}\right) \\
& =v+B_{\alpha} F_{B_{\alpha}}(j)\left(v+\left(B_{\alpha} F_{B_{\alpha}}(1)\right)^{-1}\left(b_{\alpha}\right)\right) \\
& =v+F_{B_{\alpha}}(j)\left(B_{\alpha} v+d_{\alpha}\right)=c(j) .
\end{aligned}
$$

The functional Eq. (7) for $F_{B}(t)$ implies $0=F_{B}(0)=F_{B}(-1+1)=F_{B}(-1)+$ $\exp (-B) F_{B}(1)$, hence

$$
F_{B}(-1)=-\exp (-B) F_{B}(1) ; F_{B}(-1)^{-1}=-\exp (B) F_{B}(1)^{-1} .
$$

Note that the matrices $B, F_{B}(t), F_{B}(t)^{-1}$ commute. With this identity we obtain for $j<0$ :

$$
\begin{aligned}
x_{j} & =v+\left(A_{\alpha}^{j}-\mathbb{1}\right)\left(v-\left(A_{\alpha}^{-1}-\mathbb{1}\right)^{-1}\left(A_{\alpha}^{-1} b_{\alpha}\right)\right) \\
& =v+B_{\alpha} F_{B_{\alpha}}(j)\left(v-\left(B_{\alpha} F_{B_{\alpha}}(-1)\right)^{-1} \exp \left(-B_{\alpha}\right)\left(b_{\alpha}\right)\right) \\
& =v+B_{\alpha} F_{B_{\alpha}}(j)\left(v-F_{B_{\alpha}}(-1)^{-1} B_{\alpha}^{-1} \exp \left(-B_{\alpha}\right) F_{B_{\alpha}}(1)\left(d_{\alpha}\right)\right) \\
& =v+F_{B_{\alpha}}(j)\left(B_{\alpha} v+d_{\alpha}\right)=c(j) .
\end{aligned}
$$

Remark 2. (a) Using the identification Eq. (4) we can write

$$
\left(\begin{array}{c}
x_{j+1} \\
\hline 1
\end{array}\right)=\left(\begin{array}{c|c}
A_{\alpha} & b_{\alpha} \\
\hline 0 & 1
\end{array}\right)\left(\frac{x_{j}}{1}\right) ;\left(\frac{x_{j}}{1}\right)=\left(\begin{array}{c|c}
A_{\alpha} & b_{\alpha} \\
\hline 0 & 1
\end{array}\right)^{j}\left(\frac{v}{1}\right)
$$

for all $j \in \mathbb{Z}$. 
(b) If $A_{\alpha}=\exp \left(B_{\alpha}\right)$ for a $(n, n)$-matrix $B_{\alpha}$ and if $b_{\alpha}=F_{B_{\alpha}}(1)\left(d_{\alpha}\right)$ for a vector $d_{\alpha} \in \mathbb{R}^{n}$ then we obtain from Eq. (10):

$$
\begin{aligned}
\left(\frac{c(t)}{1}\right) & =\exp \left(\left(\frac{B_{\alpha} \mid d_{\alpha}}{0}\right) t\right)\left(\frac{v}{1}\right)=\left(\begin{array}{c|c}
\exp \left(B_{\alpha} t\right) \mid F_{B_{\alpha}}(t)\left(d_{\alpha}\right) \\
0
\end{array}\right)\left(\frac{v}{1}\right) \\
& =\left(\frac{\exp \left(B_{\alpha} t\right)(v)+F_{B_{\alpha}}(t)\left(d_{\alpha}\right)}{1}\right)=\left(\frac{v+F_{B_{\alpha}}(t)\left(B_{\alpha} v+d_{\alpha}\right)}{1}\right)
\end{aligned}
$$

Hence $t \in \mathbb{R} \longmapsto c(t) \in \mathbb{R}^{n}$ is the orbit of a one-parameter subgroup of the affine group applied to the vector $v$.

\section{Smooth curves invariant under $M_{\alpha}$}

For a smooth curve $c: \mathbb{R} \longrightarrow \mathbb{R}^{n}$ and a parameter $\alpha \in(0,1)$ we define the one-parameter family $\tilde{c}_{s}: \mathbb{R} \longrightarrow \mathbb{R}^{n}, s \in \mathbb{R}$ by Eq. (2). And we call a smooth curve $c: \mathbb{R} \longrightarrow \mathbb{R}^{n}$ a soliton of the mapping $M_{\alpha}$ (resp. affinely invariant under $\left.M_{\alpha}\right)$ if there is $\epsilon>0$ and a smooth map $\in(-\epsilon, \epsilon) \longrightarrow(A, b) \in \operatorname{Aff}(n)$ such that

$$
\tilde{c}_{s}(t)=(1-\alpha) c(t)+\alpha c(t+s)=A(s)(c(t))+b(s) .
$$

Then we obtain as an analogue of $[9$, Thm.1]:

Theorem 2. Let $c: \mathbb{R} \longrightarrow \mathbb{R}^{n}$ be a soliton of the mapping $M_{\alpha}$ satisfying Eq. (14). Assume in addition that for some $t_{0} \in \mathbb{R}$ the vectors $\dot{c}\left(t_{0}\right), \ddot{c}\left(t_{0}\right), \ldots$, $c^{(n)}\left(t_{0}\right)$ are linearly independent.

Then the curve $c$ is the unique solution of the differential equation

$$
\dot{c}(t)=B c(t)+d
$$

for $B=\alpha^{-1} A^{\prime}(0), d=\alpha^{-1} b^{\prime}(0)$ with initial condition $v=c(0)$.

And $A(s)=(1-\alpha) \mathbb{1}+\alpha \exp (B s), b(s)=\alpha F_{B}(s)(d)$.

Hence the curve $c(t)$ is the orbit of a one-parameter subgroup

$$
t \in \mathbb{R} \longmapsto B(t):=\exp \left(\left(\begin{array}{c|c}
B \mid d \\
\hline 0 & 0
\end{array}\right) t\right)=\left(\exp (B t), F_{B}(t)(d)\right) \in \operatorname{Aff}(n)
$$

of the affine group, i.e.

$$
c(t)=B(t)\left(\frac{v}{1}\right)=v+F_{B}(t)(B v+d),
$$

cf. Remark 1 .

Remark 3. For an affine map $(A, b) \in \mathrm{Gl}(n), b \in \mathbb{R}^{n}$ the linear isomorphism $A$ is called the linear part. For $n=2$ we discuss the possible normal forms of $A \in \mathrm{Gl}(2)$ resp. the normal forms of the one-parameter subgroup $\exp (t B)$ and of the one-parameter family $A(s)=(1-\alpha)+\mathbb{1}+\exp (B s)$ introduced in Theorem 2. This will be used in Sect. 5 . 
1. $A=\left(\begin{array}{ll}\lambda & 0 \\ 0 & \mu\end{array}\right)$ for $\lambda, \mu \in \mathbb{R}-\{0\}$, i.e. $A$ is diagonalizable (over $\mathbb{R}$ ), then $A$ is called scaling, for $\lambda=\mu$ it is called homothety. For an endomorphism $B$ which is diagonalizable over $\mathbb{R}$ the one-parameter subgroup $B(t)=$ $\exp (B t)$ as well as the one-parameter family $A(s)=(1-\alpha) \mathbb{1}+\alpha \exp (B s)$ consists of scalings.

2. $A=\left(\begin{array}{cc}a & -b \\ b & a\end{array}\right)$ for $a, b \in \mathbb{R}, b \neq 0$, i.e. $A$ has no real eigenvalues. Then $A$ is called a similarity, i.e. a composition of a rotation and a homothety. For an endomorphism $B$ with no real eigenvalues the one-parameter subgroup $B(t)=\exp (B t), t \neq 0$ as well as the one-parameter family $A(s)=(1-$ $\alpha) \mathbb{1}+\alpha \exp (B s), s \neq 0$ consist of affine mappings without real eigenvalues, i.e. compositions of non-trivial rotations and homotheties.

3. $A=\left(\begin{array}{ll}1 & 1 \\ 0 & 1\end{array}\right)$ is called shear transformation. Hence the matrix $A$ has only one eigenvalue 1 and is not diagonalizable. If $B$ is of the form $B=$ $\left(\begin{array}{ll}0 & 1 \\ 0 & 0\end{array}\right)$, i.e. $B$ is nilpotent, then the one-parameter subgroup $B(t)=$ $\exp (B t), t \neq 0$ as well as the one-parameter family $A(s)=(1-\alpha) \mathbb{1}+$ $\alpha \exp (B s), s \neq 0$ consist of shear transformations.

4. $A=\left(\begin{array}{ll}\lambda & 1 \\ 0 & \lambda\end{array}\right)$ with $\lambda \in \mathbb{R}-\{0,1\}$. Then $A$ is invertible with only one eigenvalue $\lambda \neq 1$ and not diagonalizable. This linear map is a composition of a homothety and a shear transformation. The one-parameter subgroup $B(t)=\exp (B t), t \neq 0$ as well as the one-parameter family $A(s)=(1-\alpha) \mathbb{1}+\alpha \exp (B s), s \neq 0$ consist of linear mappings with only one eigenvalue different from 1 which are not diagonalizable. Hence they are compositions of non-trivial homotheties and shear transformations, too.

We use the following convention: For a one-parameter family $s \mapsto c_{s}$ of curves or a one-parameter family $s \mapsto A(s), s \mapsto b(s)$ of affine maps we denote the differentiation with respect to the parameter $s$ by '. On the other hand we use for the differentiation with respect to the curve parameter $t$ of the curves $t \mapsto c(t), t \mapsto c_{s}(t)$ the notation $\dot{c}, \dot{c}_{s}$.

Proof. The proof is similar to the Proof of Theorem [9, Thm.1]: Let

$$
c_{s}(t)=A(s) c(t)+b(s)=(1-\alpha) c(t)+\alpha c(t+s) .
$$

For $s=0$ we obtain $c(t)=c_{0}(t)=A(0) c(t)+b(0)$ for all $t \in \mathbb{R}$, resp. $(A(0)-\mathbb{1})(c(t))=-b(0)$ for all $t$. We conclude that

$$
(A(0)-\mathbb{1})\left(c^{(k)}(t)\right)=0
$$

for all $k \geq 1$. Since for some $t_{0}$ the vectors $\dot{c}\left(t_{0}\right), \ddot{c}\left(t_{0}\right), \ldots, c^{(n)}\left(t_{0}\right)$ are linearly independent by assumption we conclude from Eq. $(16): A(0)=\mathbb{1}, b(0)=0$. 
Eq. (15) implies for $k \geq 1$ :

$$
A(s) c^{(k)}(t)=(1-\alpha) c^{(k)}(t)+\alpha c^{(k)}(t+s)
$$

and hence

$$
A^{\prime}(s) c^{(k)}(t)=\alpha c^{(k+1)}(t+s) .
$$

We conclude from Eq. (15):

$$
\begin{aligned}
\frac{\partial c_{s}(t)}{\partial s} & =A^{\prime}(s) c(t)+b^{\prime}(s) \\
& =\frac{\partial c_{s}(t)}{\partial t}-(1-\alpha) \dot{c}(t)=(A(s)-(1-\alpha) \mathbb{1}) \dot{c}(t) .
\end{aligned}
$$

Since $A(0)=\mathbb{1}$ the endomorphisms $A(s)+(\alpha-1) \mathbb{1}$ are isomorphisms for all $s \in(0, \epsilon)$ for a sufficiently small $\epsilon>0$. Hence we obtain for $s \in(0, \epsilon)$ :

$$
\dot{c}(t)=(A(s)+(\alpha-1) \mathbb{1})^{-1} A^{\prime}(s) c(t)+(A(s)+(\alpha-1) \mathbb{1})^{-1} b^{\prime}(s) .
$$

Differentiating with respect to $s$ :

$$
\left((A(s)+(\alpha-1) \mathbb{1})^{-1} A^{\prime}(s)\right)^{\prime}(c(t))+\left((A(s)+(\alpha-1) \mathbb{1})^{-1} b^{\prime}(s)\right)^{\prime}=0
$$

and differentiating with respect to $t$ :

$$
\left((A(s)+(\alpha-1) \mathbb{1})^{-1} A^{\prime}(s)\right)^{\prime} c^{(k)}(t)=0 ; \quad k=1,2, \ldots, n .
$$

By assumption the vectors $\dot{c}\left(t_{0}\right), \ddot{c}\left(t_{0}\right), \ldots, c^{(n)}\left(t_{0}\right)$ are linearly independent. Therefore we obtain $\left((A(s)+(\alpha-1) \mathbb{1})^{-1} A^{\prime}(s)\right)^{\prime}=0$. Let $B=\alpha^{-1} A^{\prime}(0), d=$ $\alpha^{-1} b^{\prime}(0)$. Then we conclude

$$
A^{\prime}(s)=(A(s)+(\alpha-1) \mathbb{1}) B ; \quad b^{\prime}(s)=(A(s)+(\alpha-1) \mathbb{1})(d),
$$

We obtain from Eq. (17):

$$
\dot{c}(t)=B c(t)+d \text {. }
$$

Equation (18) with $A(0)=\mathbb{1}$ implies $A(s)=(1-\alpha) \mathbb{1}+\alpha \exp (B s)$. And we obtain $b^{\prime}(s)=\alpha \exp (B s)(d)=\alpha F_{B}^{\prime}(s)(d)$. Hence $b(s)=\alpha F_{B}(s)(d)$ since $b(0)=0$.

As a consequence we obtain the following

Theorem 3. For a $(n, n)$-matrix $B$ and a vector $d$ any solution of the inhomogeneous linear differential equation $\dot{c}(t)=B c(t)+d$ with constant coefficients is a soliton of the mapping $M_{\alpha}$. These solitons are orbits of a one-parameter subgroup of the affine group, i.e. they are of the form given in Eq. (9).

Proof. Any solution of the equation $\dot{c}(t)=B c(t)+d$ has the form

$$
c(t)=v+F_{B}(t)(B v+d)
$$


with $v=c(0)$, cf. Proposition 1. Then with $A(s)=(1-\alpha) \mathbb{1}+\alpha \exp (B s)$ and $b(s)=\alpha F_{B}(s)(d)$ we conclude from Eqs. (6) and (7):

$$
\begin{aligned}
\tilde{c}_{s}(t) & =(1-\alpha) c(t)+\alpha c(t+s) \\
& =v+(1-\alpha) F_{B}(t)(B v+d)+\alpha F_{B}(t+s)(B v+d) \\
& =v+(1-\alpha)(c(t)-v)+\alpha\left(F_{B}(s)+\exp (B s) F_{B}(t)\right)(B v+d) \\
& =(1-\alpha) c(t)+\alpha \exp (B s)(v)+\alpha F_{B}(s)(d)+\alpha \exp (B s)(c(t)-v) \\
& =((1-\alpha) \mathbb{1}+\alpha \exp (B s))(c(t))+\alpha F_{B}(s)(d) \\
& =A(s) c(t)+b(s) .
\end{aligned}
$$

Hence $c$ is a soliton of the mapping $M_{\alpha}$, cf. Eq. (14).

Remark 4. In [9] the authors consider the curve shortening process $T: \mathcal{P}\left(\mathbb{R}^{n}\right)$ $\longrightarrow \mathcal{P}\left(\mathbb{R}^{n}\right), T(x)_{j}=M^{2}(x)_{j-1}$ satisfying Eq. (3). Hence the midpoints mapping $M$ is applied twice followed by an index shift. The smooth curves $c=c(t)$ invariant under this process can be characterized as solutions of a inhomogeneous linear system of second order differential equations

$$
\ddot{c}(t)=B c(t)+d
$$

for a $(n, n)$-matrix $B$ and a vector $d$, cf. [9, Thm. 2]. These solutions can be reduced to a system of first order differential equations, cf. [9, Rem. 1]. Explicit formulas for these solutions can be written down in terms of power series in $t$ whose coefficients are expressed in terms of powers of $B$. If $B=B_{1}^{2}, d=B_{1}\left(d_{1}\right)$ for a $(n, n)$-matrix $B_{1}$ and a vector $d_{1} \in \mathbb{R}^{n}$, then the orbits of one-parameter subgroups of the affine group $\operatorname{Aff}\left(\mathbb{R}^{n}\right)$ acting on $\mathbb{R}^{n}$ satisfying

$$
\dot{c}(t)=B_{1} c(t)+d_{1}
$$

are particular solutions. In the next section we will see that for $n=2$ the solitons of the midpoints mapping have constant affine curvature. On the other hand not all solitons of the process $T$ have constant affine curvature, cf. $[9$, Sec. 5].

\section{Curves with constant affine curvature}

The orbits of one-parameter subgroups of the affine group Aff(2) acting on $\mathbb{R}^{2}$ can also be characterized as curves of constant general-affine curvature parametrized proportional to general-affine arc length unless they are parametrizations of a parabola, an ellipse or a hyperbola. This will be discussed in this section. The one-parameter subgroups are determined by an endomorphism $B$ and a vector $d$. We describe in Proposition 2 how the general-affine curvature can be expressed in terms of the matrix $B$.

For certain subgroups of the affine group Aff(2) one can introduce a corresponding curvature and arc length. One should be aware that sometimes in the literature the curvature related to the equi-affine subgroup $S \operatorname{Aff}(2)$ generated by the special linear group SL(2) of linear maps of determinant one and 
the translations is also called affine curvature. We distinguish in the following between the equi-affine curvature $k_{e a}$ and the general-affine curvature $k_{g a}$ as well as between the equi-affine length parameter $s_{e a}$ and the general-affine length parameter $s_{g a}$.

We recall the definition of the equi-affine and general-affine curvature of a smooth plane curve $c: I \longrightarrow \mathbb{R}^{2}$ with $\operatorname{det}(\dot{c}(t) \ddot{c}(t))=|\dot{c}(t) \ddot{c}(t)| \neq 0$ for all $t \in I$.

By eventually changing the orientation of the curve we can assume $|\dot{c}(t) \ddot{c}(t)|>$ 0 for all $t \in I$. A reference is the book by P. and A.Schirokow [10, $\S 10]$ or the recent article by Kobayashi and Sasaki [7]. Then $s_{e a}(t):=\int|\dot{c}(t) \ddot{c}(t)|^{1 / 3} d t$ is called equi-affine arc length. We denote by $t=t\left(s_{e a}\right)$ the inverse function, then $\tilde{c}\left(s_{e a}\right)=c\left(t\left(s_{e a}\right)\right)$ is the parametrization by equi-affine arc length. Then $\tilde{c}^{\prime \prime \prime}\left(s_{e a}\right), \tilde{c}^{\prime}\left(s_{e a}\right)$ are linearly dependent and the equi-affine curvature $k_{e a}\left(s_{e a}\right)$ is defined by

$$
\tilde{c}^{\prime \prime \prime}\left(s_{e a}\right)=-k_{e a}\left(s_{e a}\right) \tilde{c}^{\prime}\left(s_{e a}\right)
$$

resp.

$$
k_{e a}\left(s_{e a}\right)=\left|\tilde{c}^{\prime \prime}\left(s_{e a}\right) \tilde{c}^{\prime \prime \prime}\left(s_{e a}\right)\right| .
$$

Assume that $c=c\left(s_{e a}\right), s_{e a} \in I$ is a smooth curve parametrized by equi-affine arc length for which the $\operatorname{sign} \epsilon=\operatorname{sign}\left(k_{e a}\left(s_{e a}\right)\right) \in\{0, \pm 1\}$ of the equi-affine curvature is constant. If $\epsilon=0$ then the curve is up to an affine transformation a parabola $\left(t, t^{2}\right)$. Now assume $\epsilon \neq 0$ and let $\mathrm{K}_{e a}=\left|k_{e a}\right|=\epsilon k_{e a}$. Then the general-affine arc length $s_{g a}=s_{g a}\left(s_{e a}\right)$ is defined by

$$
s_{g a}=\int \sqrt{\mathrm{K}_{e a}\left(s_{e a}\right)} d s_{e a} .
$$

We call a curve $c=c(t)$ parametrized proportional to general-affine arc length if $t=\lambda_{1} s_{g a}+\lambda_{2}$ for $\lambda_{1}, \lambda_{2} \in \mathbb{R}$ with $\lambda_{1} \neq 0$. The general-affine curvature $k_{g a}=k_{g a}\left(s_{e a}\right)$ is defined by

$$
k_{g a}\left(s_{e a}\right)=\mathrm{K}_{e a}^{\prime}\left(s_{e a}\right) \mathrm{K}_{e a}\left(s_{e a}\right)^{-3 / 2}=-2\left(\mathrm{~K}_{e a}^{-1 / 2}\left(s_{e a}\right)\right)^{\prime} .
$$

If the general-affine curvature $k_{g a}$ (up to sign) and the sign $\epsilon$ is given with respect to the equi-affine arc length parametrization, then the equi-affine curvature $k_{e a}=k_{e a}\left(s_{e a}\right)$ is determined up to a constant by Eq. (20). Hence the curve is determined up to an affine transformation. The invariant $k_{g a}$ already occurs in Blaschke's book [2, 110, p.24]. Curves of constant general-affine curvature are orbits of a one-parameter subgroup of the affine group. These curves already were discussed by Klein and Lie [6] under the name $W$-curves.

Proposition 2. For a non-zero matrix $B \in M_{\mathbb{R}}(2,2)$ and vectors $d, v \in \mathbb{R}^{2}$ where $B v+d$ is not an eigenvector of $B$ let $c: \mathbb{R} \longrightarrow \mathbb{R}^{2}$ be the solution of the differential equation $c^{\prime}(t)=B c(t)+d ; c(0)=v$, i.e. $c(t)=v+$ $F_{B}(t)(B v+d)=\exp (t B)(v)+F_{B}(t)(d)$. We assume that $\beta=\left|c^{\prime}(0) c^{\prime \prime}(0)\right|^{1 / 3}=$ $|B v+d B(B v+d)|^{1 / 3}>0$. Define 


$$
k=k(B)=-2+9 \operatorname{det}(B) / \operatorname{tr}^{2}(B) ; \quad K=K(B)=|k(B)|^{-1 / 2} .
$$

(a) If $\operatorname{tr}(B)=0$ then the curve is parametrized proportional to equi-affine arc length and the equi-affine curvature is constant $k_{e a}=\operatorname{det}(B) / \beta^{2}$ and $\epsilon=$ $\operatorname{sign}(\operatorname{det}(B))$, the curve is a parabola, if $\epsilon=0$, an ellipse, if $\epsilon>0$, or a hyperbola, if $\epsilon<0$, cf. Remark 5.

(b) If $\operatorname{tr}(B) \neq 0$ then we can choose a parametrization by equi-affine arc length $s_{e a}$ such that the equi-affine curvature $k_{e a}$ is given by:

$$
k_{e a}\left(s_{e a}\right)=k(B) s_{e a}{ }^{-2} .
$$

If $k(B)=0$ the curve has vanishing equi-affine curvature and is a parametrization of a parabola, cf. the Remark 5 . If $k(B) \neq 0$ then the general-affine curvature is defined and constant:

$$
k_{g a}\left(s_{e a}\right)=-2 K(B) .
$$

Up to an additive constant the general-affine arc length parameter $s_{g a}$ is given by:

$$
s_{g a}=\frac{\operatorname{tr} B}{3 K(B)} t .
$$

Hence the curve $c(t)$ is parametrized proportional to general-affine arc length.

Remark 5. It is well-known that the curves of constant equi-affine curvature are parabola, hyperbola or ellipses, cf. [2, $\S 7]$. For $k_{e a}=0$ we obtain a parabola: $c(t)=c(0)+c^{\prime}(0) s_{e a}+c^{\prime \prime}(0) s_{e a}^{2} / 2$, for $k_{e a}>0$ the ellipse $c\left(s_{e a}\right)=$ $\left(a \cos \left(\sqrt{k_{e a}} s_{e a}\right), b \sin \left(\sqrt{k_{e a}} s_{e a}\right)\right)$ with $k_{e a}=(a b)^{-2 / 3}$ and for $k_{e a}<0$ the hyperbola $c\left(s_{e a}\right)=\left(a \cosh \left(\sqrt{-k_{e a}} s_{e a}\right), b \sinh \left(\sqrt{-k_{e a}} s_{e a}\right)\right)$ with $k_{e a}=-(a b)^{-2 / 3}$. Here $a, b>0$.

Proof. Following Proposition 1 we obtain as solution of the differential equation: $c(t)=v+F_{B}(t)(B v+d)$, hence for the derivatives: $c^{(k)}(t)=B^{k-1} \exp (t B)$ $(B v+d)$. Then :

$$
\begin{aligned}
|\dot{c}(t) \ddot{c}(t)| & =|\exp (B t)||b v+d B(B v+d)| \\
& =\exp (\operatorname{tr}(B) t)|B v+d B(B v+d)| .
\end{aligned}
$$

Let $\beta=(|B v+d B(B v+d)|)^{1 / 3}$ and $\tau=\operatorname{tr}(B)$. Then

$$
|\dot{c}(t) \ddot{c}(t)|=\beta^{3} \exp (\tau t) .
$$

(a) If $\tau=0$ then $s_{e a}=t \beta$, i.e. the curve is parametrized proportional to equi-affine arc length and

$$
\tilde{c}\left(s_{e a}\right)=c\left(t\left(s_{e a}\right)\right)=c\left(s_{e a} / \beta\right)=v+F_{B}\left(s_{e a} / \beta\right)(B v+d) .
$$

Then

$$
\begin{aligned}
\tilde{c}^{\prime}\left(s_{e a}\right) & =\beta^{-1} \exp \left(B s_{e a} / \beta\right)(B v+d) \\
\tilde{c}^{\prime \prime \prime}\left(s_{e a}\right) & =\beta^{-3} B^{2} \exp \left(B s_{e a} / \beta\right)(B v+d)=-\operatorname{det}(B) \beta^{-2} \tilde{c}^{\prime}\left(s_{e a}\right) .
\end{aligned}
$$


Here we use that by Cayley-Hamilton $B^{2}-\tau B=B^{2}=-\operatorname{det}(B) \cdot \mathbb{1}$. Hence we obtain $k_{e a}\left(s_{e a}\right)=\operatorname{det}(B) / \beta^{2}$ and $\epsilon=\operatorname{sign}(\operatorname{det}(B))$. Then the claim follows from Remark 5 .

(b) Assume $\tau \neq 0$. Then the equi-affine arc length $s_{e a}=s_{e a}(t)$ is given by

$$
s_{e a}(t)=\beta \int \exp (\tau t / 3) d t=\frac{3 \beta}{\tau} \exp (\tau t / 3) .
$$

Hence the equi-affine arc length parametrization of $c$ is given by

$$
\tilde{c}\left(s_{e a}\right)=v+F_{B}\left(\frac{3}{\tau} \ln \left(\frac{\tau}{3 \beta} s_{e a}\right)\right)(B v+d) .
$$

Then we can express the derivatives:

$$
\begin{aligned}
\tilde{c}^{\prime}\left(s_{e a}\right) & =\frac{3}{\tau} \frac{1}{s_{e a}} \exp \left(\frac{3}{\tau} B \ln \left(\frac{\tau}{3 \beta} s_{e a}\right)\right)(B v+d) \\
\tilde{c}^{\prime \prime}\left(s_{e a}\right) & =\left(\frac{3}{\tau} B-\mathbb{1}\right) \frac{1}{s_{e a}} \tilde{c}^{\prime}\left(s_{e a}\right) \\
\tilde{c}^{\prime \prime \prime}\left(s_{e a}\right) & =\left(\frac{3}{\tau} B-\mathbb{1}\right)\left(\frac{3}{\tau} B-2 \mathbb{1}\right) \frac{1}{s_{e a}^{2}} \tilde{c}^{\prime}\left(s_{e a}\right) \\
& =-\left(\frac{9 \operatorname{det}(B)}{\tau^{2}}-2\right) \frac{1}{s_{e a}^{2}} \tilde{c}^{\prime}\left(s_{e a}\right) .
\end{aligned}
$$

Here we used that by Cayley-Hamilton $B^{2}-\tau B=-\operatorname{det} B \cdot \mathbb{1}$. Hence we obtain for the equi-affine curvature

$$
k_{e a}\left(s_{e a}\right)=\frac{k(B)}{s_{e a}^{2}} .
$$

Then $\epsilon=\operatorname{sign} k(B)$ and for $k(B) \neq 0$ we obtain from Eqs. (20) and (25):

$$
k_{g a}\left(s_{e a}\right)=-\frac{2}{K(B)} .
$$

And for the general-affine arc length we obtain

$$
s_{g a}=\ln \left(\left|s_{e a}\right|\right) / K(B),
$$

resp. up to an additive constant:

$$
s_{g a}=\frac{\tau t}{3 K(B)}
$$

using Eq. (24).

The parametrization by general-affine arc length is given by

$$
c^{*}\left(s_{g a}\right)=v+F_{B}\left(3 K(B) s_{g a} / \tau\right)(B v+d) .
$$

Example 1. Depending on the real Jordan normal forms of the endomorphism $B$ we investigate the solitons $c(t)$, their special and general affine curvature. The normal forms of the corresponding one-parameter subgroup $B(t)=\exp (B t)$ as well of the one-parameter family $A(s)=(1-\alpha) \mathbb{1}+\exp (B s)$ follow from 
Remark 3. Since $c(\mu t)=\exp (\mu B t)$ the multiplication of $B$ with a non-zero real $\mu$ corresponds to a linear reparametrization of the curve. If $B$ has a non-zero real eigenvalue we can assume without loss of generality that it is 1 and in the case of a non-real eigenvalue we can assume that it has modulus 1 .

(a) Let $B=\left(\begin{array}{ll}1 & 0 \\ 0 & \lambda\end{array}\right), d=(0,0), c(0)=(1,1)$ and $\lambda \neq 0,1$. Then $\beta=$ $(\lambda(1-\lambda))^{1 / 3} \neq 0, \operatorname{tr} B=1+\lambda$ and $c(t)=(\exp (t), \exp (\lambda t))$. Up to parametrization we have $c(u)=\left(u, u^{\lambda}\right)$.

If $\lambda=-1$ then $c$ is a parametrization of a hyperbola, $\operatorname{tr} B=0$ and $k_{e a}=-2^{-2 / 3}$, cf. Remark 5 .

If $\lambda \neq-1$ we obtain for the equi-affine curvature with respect to a equi-affine parametrization $s_{e a}$ from Eq. (22):

$$
k_{e a}\left(s_{e a}\right)=\left(9 \frac{\operatorname{det} B}{\operatorname{tr}^{2} B}-2\right) \frac{1}{s_{e a}^{2}}=-\frac{(\lambda-2)(2 \lambda-1)}{(\lambda+1)^{2}} \frac{1}{s_{e a}^{2}} .
$$

For $\lambda=1 / 2,2$ we obtain a parametrization of a parabola with vanishing equi-affine curvature, cf. Remark 5 . Now we assume $\lambda \neq 1 / 2,2$. Hence $\epsilon=1$ if and only if $1 / 2<\lambda<2$. The affine curvature $k_{g a}$ is constant:

$$
k_{g a}=-2 \frac{|\lambda+1|}{\sqrt{|(\lambda-2)(2 \lambda-1)|}},
$$

cf. [7, Ex.2.14]. We have $\epsilon=1$ if and only if $1 / 2<\lambda<2$, then $k_{g a} \in(-\infty,-4)$. And $\epsilon=-1$ if and only if $\lambda<1 / 2, \lambda \neq 0$ or $\lambda>2$, then $k_{g a} \in(-\infty,-\sqrt{2}) \cup(-\sqrt{2}, 0)$.

Hence in this case the corresponding one-parameter subgroup

$$
B(t)=\left(\begin{array}{cc}
\exp (t) & 0 \\
0 & \exp (\lambda t)
\end{array}\right)
$$

as well as the one-parameter family

$$
A(s)=\left(\begin{array}{cc}
1-\alpha+\alpha \exp (s) & 0 \\
0 & 1-\alpha+\alpha \exp (\lambda s)
\end{array}\right)
$$

consist of scalings.

(b) $B=\left(\begin{array}{ll}0 & 0 \\ 0 & 1\end{array}\right)$ and $d=(1,0)$. Then the solution of the Equation $\dot{c}(t)=$ $B c(t)+d$ with $c(0)=(0,1)$ is of the form $c(t)=(t, \exp (t))$. Then we obtain $\epsilon=-1$ and $k_{g a}=-\sqrt{2}$. The corresponding one-parameter subgroup $B(t)$ as well as the one-parameter family $A(s)$ consist of scalings, the affine transformation $(A(s), b(s)$ is given by $(A(s), b(s))=$ $\left(\left(\begin{array}{lc}1 & 0 \\ 0 & 1-\alpha+\alpha \exp (s)\end{array}\right), \alpha\left(\begin{array}{l}s \\ 0\end{array}\right)\right)$

i.e. a composition of scalings and translations. 


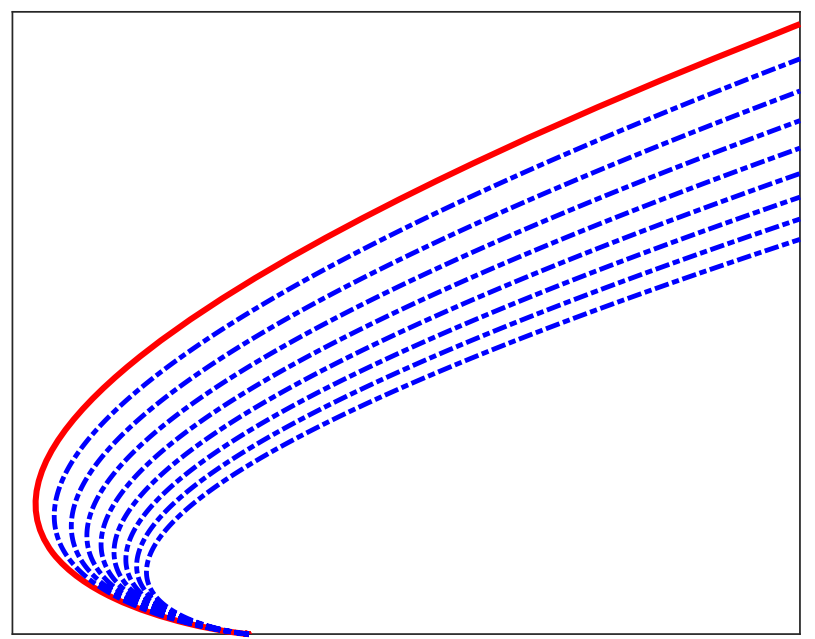

Figure 2 The soliton $c(t)=((t+1) \exp (t), \exp (t))$ with the family $c_{s}(t)=A(s) c(t)$

(c) If $B=\left(\begin{array}{ll}1 & 1 \\ 0 & 1\end{array}\right), d=(0,0), c(0)=(1,1)$ then $c(t)=((t+1) \exp (t), \exp (t))$ i.e. up to an affine transformation and a reparametrization the curve is of the form $c(u)=(u, u \ln (u))$. Then $\epsilon=1$ and $k_{g a}=-4$. The corresponding one-parameter subgroup $B(t)$ as well as the one-parameter family $A(s)$ consist of compositions of a homothety and a shear transformations;

$$
B(t)=\exp (t) \cdot\left(\begin{array}{cc}
1 & t \\
0 & 1
\end{array}\right) ; A(s)=\left(\begin{array}{cc}
1-\alpha+\alpha \exp (s) & \alpha s \exp (s) \\
0 & 1-\alpha+\alpha \exp (s)
\end{array}\right),
$$

cf. Fig. 2.

(d) If $B=\left(\begin{array}{rr}a & -b \\ b & a\end{array}\right)$ with $b \neq 0, a^{2}+b^{2}=1, d=0, c(0)=(1,0)$ then $c(t)=$ $\exp (a t)(\cos (b t), \sin (b t))$. For $a=0$, this is a circle with $k_{e a}=1$. Now we assume $a \neq 0$ : Then $\epsilon=\operatorname{sign}\left(9 \operatorname{det}(B)-2 \operatorname{tr}^{2}(B)\right)=\operatorname{sign}\left(a^{2}+9 b^{2}\right)=1$ and we obtain for the general-affine curvature $k_{g a}=-4|a| / \sqrt{a^{2}+9 b^{2}}=$ $-4|a| / \sqrt{9-8 a^{2}}$, i.e. $k_{g a} \in(-4,0)$. The corresponding one-parameter subgroup $B(t)$ as well as the one-parameter family $A(s)$ consist of similarities.

(e) If $B=\left(\begin{array}{ll}0 & 1 \\ 0 & 0\end{array}\right)$ one can choose $c(0)=(0,0), d=(0,1)$ and obtain $c(t)=\left(t^{2} / 2, t\right)$, i.e. a parabola. In this case the one parameter subgroup $B(t)=\exp (t B)$ consists of shear transformations. The one-parameter family $(A(s), b(s))=\left(\left(\begin{array}{cc}1 & \alpha s \\ 0 & 1\end{array}\right), \alpha\left(\begin{array}{c}s^{2} / 2 \\ s\end{array}\right)\right)$ consists of a composition of a shear transformation and a translation, cf. Fig. 1. For the affine curve shortening flow the parabola is a translational soliton. Therefore it is also called the affine analogue of the grim reaper, cf. [3, p. 192]. For the curve 
shortening process $T$ defined by Eq. (3) the parabola is also a translational soliton, cf. [9, Sec. 5, Case (5)].

Note that the parabola occurs twice, in Case (a) it occurs with the parametrization $c(t)=(\exp (t), \exp (2 t))$, in Case (e) it occurs with a parametrization proportional to equi-affine arc length. Summarizing we obtain from Theorems 2 and 3 together with Proposition 2 resp. Example 1 the following

Theorem 4. Let $c: \mathbb{R} \longrightarrow \mathbb{R}^{2}$ be a smooth curve for which $\dot{c}(0), \ddot{c}(0)$ are linearly independent. Then $c$ is a soliton of the mappings $M_{\alpha}, \alpha \in(0,1)$, in particular of the midpoints mapping $M=M_{1 / 2}$, if it is a curve of constant equi-affine curvature parametrized proportional to equi-affine arc length, or a parabola with the parametrization $c(t)=(\exp (t), \exp (2 t))$ up to an affine transformation, or if it is a curve of constant general-affine curvature parametrized proportional to general-affine arc length.

Funding Open Access funding enabled and organized by Projekt DEAL.

Open Access. This article is licensed under a Creative Commons Attribution 4.0 International License, which permits use, sharing, adaptation, distribution and reproduction in any medium or format, as long as you give appropriate credit to the original author(s) and the source, provide a link to the Creative Commons licence, and indicate if changes were made. The images or other third party material in this article are included in the article's Creative Commons licence, unless indicated otherwise in a credit line to the material. If material is not included in the article's Creative Commons licence and your intended use is not permitted by statutory regulation or exceeds the permitted use, you will need to obtain permission directly from the copyright holder. To view a copy of this licence, visit http://creativecommons. org/licenses/by/4.0/.

Publisher's Note Springer Nature remains neutral with regard to jurisdictional claims in published maps and institutional affiliations.

\section{References}

[1] Berlekamp, E.R., Gilbert, E.N., Sinden, F.W.: A polygon problem. Am. Math. Mon. 72, 233-241 (1965)

[2] Blaschke, W.: Vorlesungen über Differentialgeometrie I. 2. Auflage (Grundlehren der math. Wiss. in Einzeldarstellungen, Bd. I). Verlag J. Springer, Berlin (1924)

[3] Calabi, E., Olver, P.J., Tannenbaum, A.: Affine geometry, curve flows, and invariant numerical approximations. Adv. Math. 124, 154-196 (1996)

[4] Darboux, G.: Sur un problème de géométrie élémentaire. Bull. Sci. Math. Astron. 2e série 2, 298-304 (1878)

[5] Glickenstein, D., Liang, J.: Asymptotic behaviour of $\beta$-polygon flows. J. Geom. Anal. 28, 2902-2952 (2018) 
[6] Klein, F., Lie, S.: Ueber diejenigen ebenen Curven, welche durch ein geschlossenes System von einfach unendlich vielen vertauschbaren linearen Transformationen in sich uebergehen. Math. Ann. 4, 50-84 (1871)

[7] Kobayashi, S., Sasaki, T.: General-affine invariants of plane curves and space curves. Czechoslov. Math. J. 70(145), 67-104 (2020)

[8] Kühnel, W.: Matrizen und Lie-Gruppen. Vieweg Teubner Verlag (2011)

[9] Rademacher, C., Rademacher, H.B.: Solitons of discrete curve shortening. Res. Math. 71, 455-482 (2017)

[10] Schirokov, P.A., Schirokov, A.P.: Affine Differentialgeometrie. Teubner Verlag, Leipzig (1962)

[11] Viera, E., Garcia, R.: Asymptotic behaviour of the shape of planar polygons by linear flows. Lin. Algebra Appl. 557, 508-528 (2018)

Christine Rademacher

Fakultät Angewandte Mathematik, Physik und Allgemeinwissenschaften, Technische Hochschule Nürnberg Georg Simon Ohm

Postfach 210320

90121 Nürnberg

Germany

e-mail: christine.rademacher@th-nuernberg.de

Hans-Bert Rademacher

Mathematisches Institut

Universität Leipzig

04081 Leipzig

Germany

e-mail: hans-bert.rademacher@math.uni-leipzig.de

Received: August 25, 2020.

Revised: December 14, 2020.

Accepted: December 22, 2020. 